\title{
Shear bands and cracking of metallic glass plates in bending
}

\author{
R. D. Conner and W. L. Johnson \\ Materials Science Group, Keck Laboratory, California Institute of Technology, Pasadena, California 91125 \\ N. E. Paton \\ Liquidmetals Technologies, Lake Forest, California 92630 \\ W. D. Nix ${ }^{\mathrm{a})}$ \\ Department of Materials Science and Engineering, Stanford University, Stanford, California 94305-2205
}

(Received 17 February 2003; accepted 24 April 2003)

\begin{abstract}
The thickness dependence of yielding and fracture of metallic glass plates subjected to bending is considered in terms of the shear band processes responsible for these properties. We argue that the shear band spacing (and length) scales with the thickness of the plate because of strain relaxation in the vicinity of the shear band at the surface. This is consistent with recent measurements of shear band spacing versus sample size. We also argue that the shear displacements in the shear band scale with the shear band length and plate thickness, thus causing cracks to be initiated in thicker plates at smaller bending strains. This leads to fracture bending strains that decrease markedly with increasing plate thickness, consistent with recent experiments. These results suggest that amorphous metals in the form of foams might have superior ductility and toughness. (C) 2003 American Institute of Physics. [DOI: 10.1063/1.1582555]
\end{abstract}

\section{INTRODUCTION}

It is commonly observed that thin ribbons or wires of amorphous metals can be bent plastically without breaking, while thick plates of the same materials fracture almost immediately on bending. Many reports can be found in the literature of thin wires or foils of amorphous metals having good ductility in bending. ${ }^{1-8}$ Katuya et al. ${ }^{9}$ and Inoue et al. ${ }^{10}$ found that significant bend ductility could be achieved only if the sample dimension was below a critical value, suggesting a size effect for bend ductility.

The observation that thin wires of metallic glasses can be bent plastically while thick plates, without surface cracks, cannot, is not expected for elastic, perfectly plastic materials subjected to bending, as this mode of deformation, unlike tension, is inherently stable. Thus, metallic glasses, with compressive deformation properties showing elastic, perfectly plastic behavior, might be expected to deform stably in bending, regardless of the plate thickness. Yet thick plates of metallic glasses invariably fracture in bending while thin ribbons deform extensively. We wish to understand this sample size effect, as it could be of importance in the development of ductile metallic glass alloys.

In the present article, we attempt to rationalize these observations by considering the deformation of thin plates of metallic glasses subjected to pure bending. We focus our attention on the plastic zones that develop at the surfaces of plates subjected to bending and on the shear bands in those plastic zones. We argue that the effect of plate thickness on the deformation and fracture properties of metallic glasses in bending can be understood in terms of the shear bands that develop during plastic flow and the transformation of those

\footnotetext{
a)Electronic mail: nix @ stanford.edu
}

shear bands into cracks. This analysis attempts to explain the observation that the shear band spacing scales with the thickness of the plate being bent. We do so by noting that the operation of a single shear band at the surface leads to unloading in the vicinity of that shear band, causing other shear bands to be excluded from that vicinity and leading to a shear band spacing that increases with increasing plate thickness. The shear offsets associated with each shear band must also scale with the length of the shear band, thus increasing with plate thickness. Therefore, because the shear bands are more widely spaced in thicker plates, and the shear offsets are larger, crack initiation in the shear bands is expected to occur more readily in thicker plates. The cracks, so initiated, can also grow more quickly in thicker plates. When they reach a critical length they propagate in an unstable manner and cause fracture to occur. Thus the bend ductility is predicted to decrease with increasing plate thickness because the shear band spacings and shear offsets are larger in thicker plates, leading to crack formation and propagation and fracture.

\section{EXPERIMENTAL OBSERVATIONS}

We have recently studied the effect of sample size on both the shear band spacing and the bend ductility of amorphous plates, ribbons and wires. Figure 1(a), is a scanning electron microscopy (SEM) micrograph of shear bands in a $0.5 \mathrm{~mm}$ thick plate, machined from a cast sample of Vitreloy 106 , bent over a mandrel with a radius of $1 \mathrm{~mm}$. The shear displacements associated with the shear bands are easily visible and secondary shear bands are also observed. The shear offsets associated with shear bands are not cracks. Figure 1(b) shows another, slightly thicker, plate of Vitreloy 106, bent over a mandrel with a radius of $1 \mathrm{~mm}$. Here, one of the shear bands appears to be developing as a crack. 


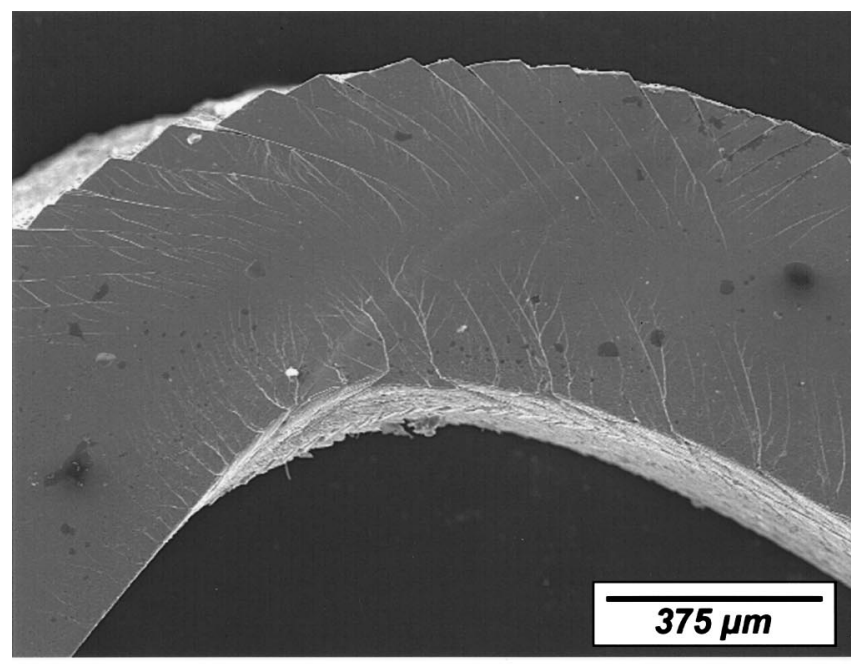

(a)

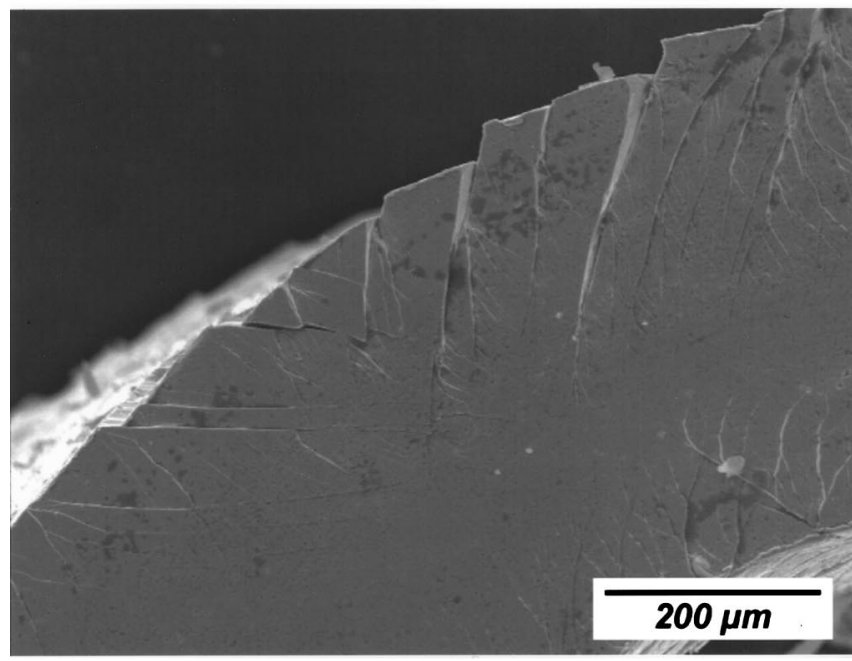

(b)

FIG. 1. (a) SEM micrograph showing shear bands in a $0.5 \mathrm{~mm}$ thick meltspun ribbon of Vitreloy 106, bent over a mandrel with a radius of $1 \mathrm{~mm}$. The shear displacements associated with the shear bands are easily visible and secondary shear bands are also observed. The shear bands on the tension side of the sample extend farther into the sample than do those on the compression side. Conner $e t$ al. ${ }^{11}$ (b) SEM micrograph showing shear bands in a $0.58 \mathrm{~mm}$ thick melt-spun ribbon of Vitreloy 106, bent over a mandrel with a radius of $1 \mathrm{~mm}$. Some of the shear bands on the tension side of the sample appear to have developed as cracks. Conner et al. ${ }^{11}$

We have also shown that the plastic strain to fracture for metallic glass wires and foils increases with decreasing sample dimension. By bending wires or strips of different thicknesses around mandrels of different radii and noting the surface strain at which failure occurs, we found that the strain to fracture increases markedly as the wire diameter drops below about $1 \mathrm{~mm}$. Figure 2 shows that the fracture strain varies approximately inversely with the square of the sample dimension.

We have also noted that the shear band spacing on the surface of the bent wires and plates scales linearly with the sample dimension, as shown in Fig. 3. There, the shear band spacing is observed to be about one tenth of the sample thickness over a wide range of sample sizes. This, too, is an important sample size effect which, we believe, leads to the

\section{Plastic Strain to Failure of Strips \& Wires}

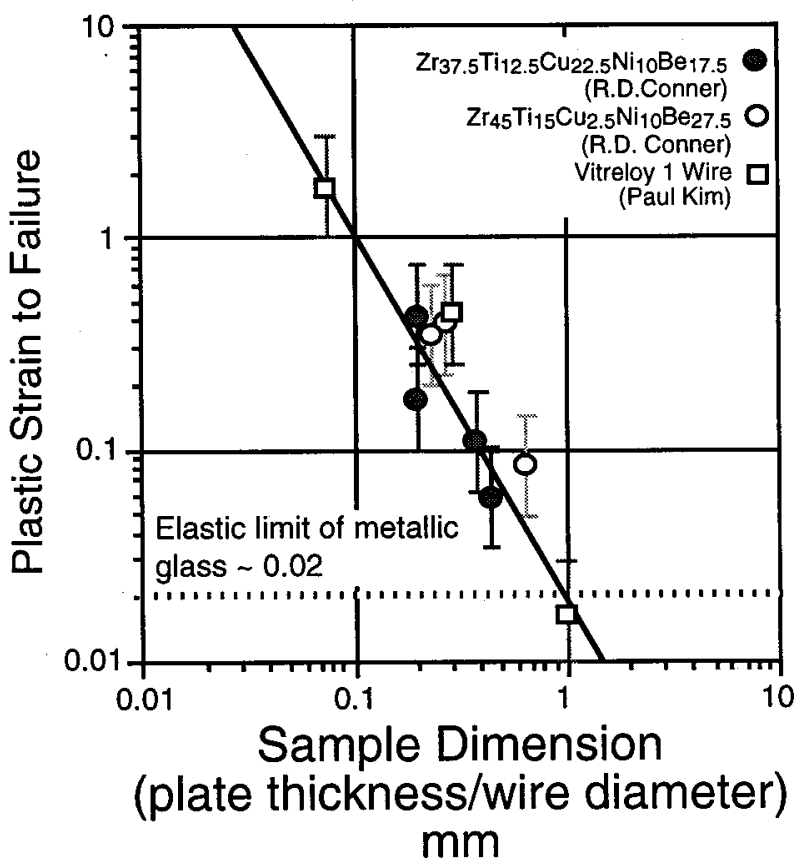

FIG. 2. Bending fracture strain vs sample dimension for various metallic glasses. The fracture strain increases dramatically with decreasing sample size below a thickness of about $1 \mathrm{~mm}$ and varies approximately inversely with the square of the sample dimension. Conner et al. ${ }^{11}$

strong effect of sample size on bend ductility. All of these experimental findings will be described in more detail in a forthcoming paper. ${ }^{11}$

\section{ELASTIC PERFECTLY PLASTIC BENDING ANALYSIS}

Consider a metallic glass plate of thickness $2 h$, deformed in plane strain bending to a curvature $\kappa$, as shown in Fig. 4. For convenience we will call the curvature shown as positive; the coordinate system will be fixed to the middle of the plate, as shown. With this notation, the strain in the plate may be expressed as

$$
\varepsilon_{x x}=\kappa\left(y-y_{0}\right),
$$

\section{Shear Band Spacing vs. Sample Dimension Metallic Glass Ribbons, Wires, Strips and Plates}

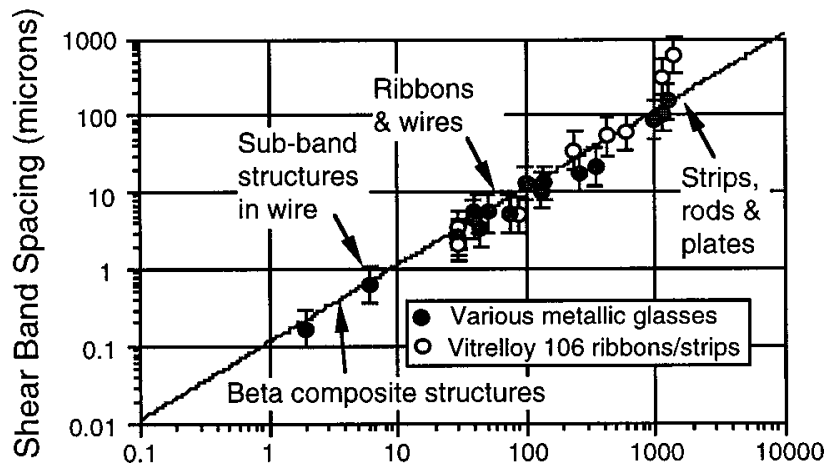

FIG. 3. Shear band spacing as a function of sample size (plate thickness or wire diameter) at the point of fracture for various metallic glasses and Vitreloy 106 . The shear band spacing is shown to scale linearly with the sample size. Conner et al. ${ }^{11}$ 


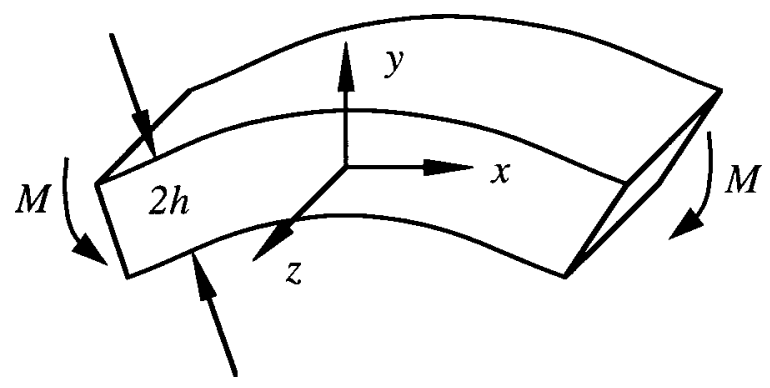

FIG. 4. Plane strain bending of a plate.

where $y_{0}$ is the position of the neutral plane for bending. Before yielding starts, the neutral plane resides in the middle of the plate, so $y_{0}=0$. It remains at that place for the case of symmetric bending, where yielding occurs equally on the top and bottom faces of the plate, but it moves during nonsymmetric bending, as discussed below.

Plastic yielding will commence at the surfaces of the plate, where the stresses are highest, when the stress there reaches the yield stress $\sigma_{y}$. This corresponds to a yield strain given by

$$
\varepsilon_{y}=\frac{(1-\nu)}{2 \mu} \sigma_{y},
$$

and a yield curvature given by

$$
\kappa_{y}=\frac{(1-\nu)}{2 \mu} \frac{\sigma_{y}}{h},
$$

where $\mu$ is the elastic shear modulus and $\nu$ is Poisson's ratio for the glass. In the present treatment we consider two different models for yielding of the plate in bending. In one model we assume that yielding occurs equally at the top and bottom surfaces of the plate. We call this the case of symmetric bending. In another model we assume that yielding occurs only on the tension side of the plate; the surface subjected to compression is assumed to deform elastically in bending. We call this the case of nonsymmetric bending. Although large tension/compression yielding asymmetries have not been reported for metallic glasses, normal stress effects on yielding suggest that the yield strength in tension might be slightly smaller than that for compression. ${ }^{12-14}$ Also, the shear bands in Fig. 1 show some evidence of a tension/ compression asymmetry; the shear bands on the tension side of the sample extend farther into the sample than do those on the compression side. In the development that follows, the relations describing each of these two models are developed in parallel.

\section{Symmetric Bending}

When the imposed curvature and moment exceeds that for yielding, plastic zones are expected to develop at the top and bottom surfaces of the plate. Using the model of the elastic, perfectly plastic solid, we may estimate the size of these plastic zones by noting the stress distributions shown in Fig. 5. Using the stress distribution in the elastic part of the plate

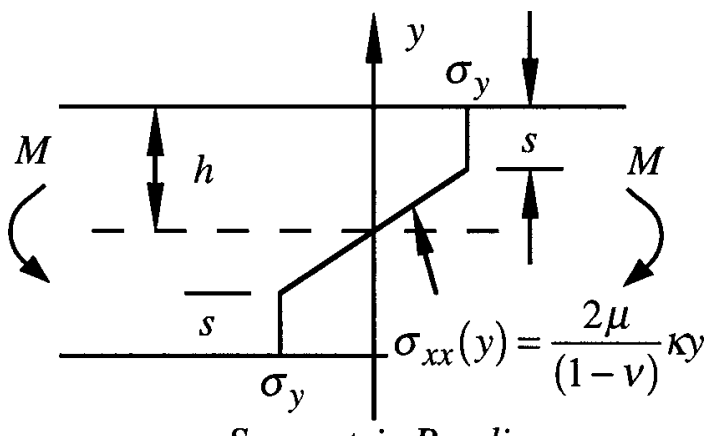

Symmetric Bending

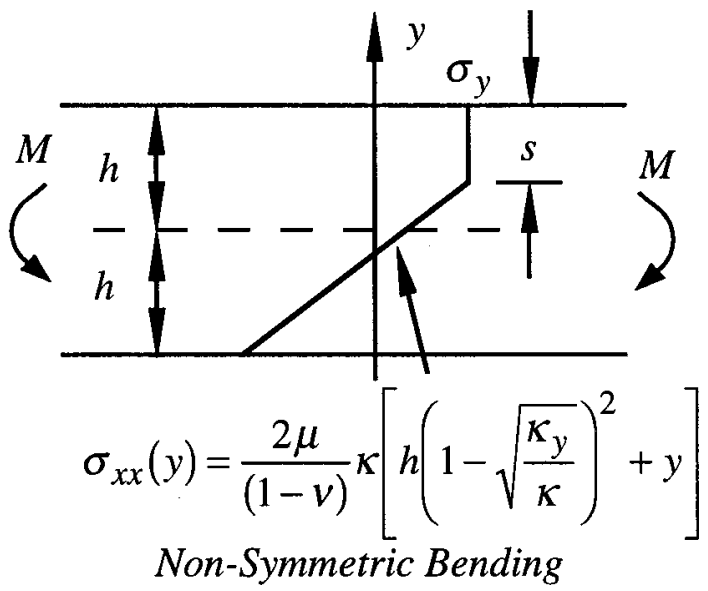

FIG. 5. Stress distribution in symmetric and nonsymmetric bending.

$$
\sigma_{x x}(y)=\frac{2 \mu}{(1-\nu)} \kappa y,
$$

as illustrated in Fig. 5, the moment (per unit width) for bending to an arbitrary curvature $\kappa$ may be expressed as

$$
\begin{aligned}
& M=2 \int_{0}^{h} \sigma_{x x} y d y, \\
& M=2 \int_{0}^{h-s} \frac{2 \mu}{(1-\nu)} \kappa y^{2} d y+2 \int_{h-s}^{h} \sigma_{y} y d y, \\
& M=\frac{2}{3} \frac{2 \mu}{(1-\nu)} \kappa(h-s)^{3}+\sigma_{y}\left(2 h s-s^{2}\right) .
\end{aligned}
$$

The plastic zone size $s$ may be found by setting the bending stress in Eq.(4) equal to $\sigma_{y}$ at $y=h-s$, such that

$$
s=h-\frac{(1-\nu)}{2 \mu} \frac{\sigma_{y}}{\kappa}=h\left(1-\frac{\kappa_{y}}{\kappa}\right) .
$$

Substituting Eq. (6) into Eq.(5) leads to the following expression for the bending moment:

$$
M=\frac{M_{y}}{2}\left[3-\left(\frac{\kappa_{y}}{\kappa}\right)^{2}\right],
$$

where

$$
M_{y}=\frac{2}{3} \frac{2 \mu}{(1-\nu)} \kappa_{y} h^{3}=\frac{2}{3} \sigma_{y} h^{2}
$$

is the yielding moment. We note that the moment described by Eq. (7) increases monotonically with increasing curvature, 
indicating that elastic/plastic bending of the kind considered here is a stable form of deformation. If cracking processes do not occur, bending deformation should be perfectly stable.

\section{Nonsymmetric Bending}

We also consider nonsymmetric bending, wherein yielding occurs in tension but not compression. Figure 5 shows the stress distribution in the plate for this kind of bending. For nonsymmetric bending, the linear stress distribution in the elastic part of the bending plate can be found by requiring the total in-plane force acting in the plate to be zero. The result is

$$
\sigma(y)=\sigma_{y}\left[1-\frac{4 h(h-s-y)}{(2 h-s)^{2}}\right] .
$$

The position of the neutral plane $y_{0}$ can be found by requiring the stress there to be zero, $\sigma\left(y=y_{0}\right)=0$. The result is

$$
y_{0}=-\frac{s^{2}}{4 h} \text {. }
$$

In analogy with Eq. (6), we may find the size of the plastic zone in terms of the imposed curvature by requiring the strain distribution in the plate to be given by $\varepsilon(y)=\kappa(y$ $\left.-y_{0}\right)$. Using Eqs. (2), (3), and (10), we can then write

$$
s=2 h\left(1-\sqrt{\frac{\kappa_{y}}{\kappa}}\right) .
$$

Inserting this relation into Eq. (9) and using Eq. (3), we find the stress distribution in the elastic part of the plate to be

$$
\sigma(y)=\frac{2 \mu}{(1-\nu)} \kappa\left[h\left(1-\sqrt{\frac{\kappa_{y}}{\kappa}}\right)^{2}+y\right],
$$

as illustrated in Fig. (5). Finally, the bending moment can be expressed as

$$
M=\int_{-h}^{h-s} \sigma(y)\left(y-y_{0}\right) d y+\int_{h-s}^{h} \sigma_{y}\left(y-y_{0}\right) d y
$$

which, with Eqs. (10)-(13), becomes

$$
M=M_{y}\left(3-2 \sqrt{\frac{\kappa_{y}}{\kappa}}\right),
$$

in analogy with Eq. (7) for the case of symmetric bending. We again note that the moment increases monotonically with increasing curvature, as expected, so that the deformation is inherently stable if cracking processes do not occur.

\section{SHEAR BAND PLASTICITY}

The analyses described above are based on the model of an elastic, perfectly plastic solid. Although metallic glasses deformed in compression nominally exhibit this kind of law, on the microscopic scale plasticity is quite different. Plastic deformation in metallic glasses is highly localized in shear bands. In the present treatment we will assume that the elastic, perfectly plastic law can be used to estimate the lengths of the shear bands at a given point in the bending process. Figure 6 shows how we might envision this highly localized plastic process. Shear bands are assumed to exist on inclined
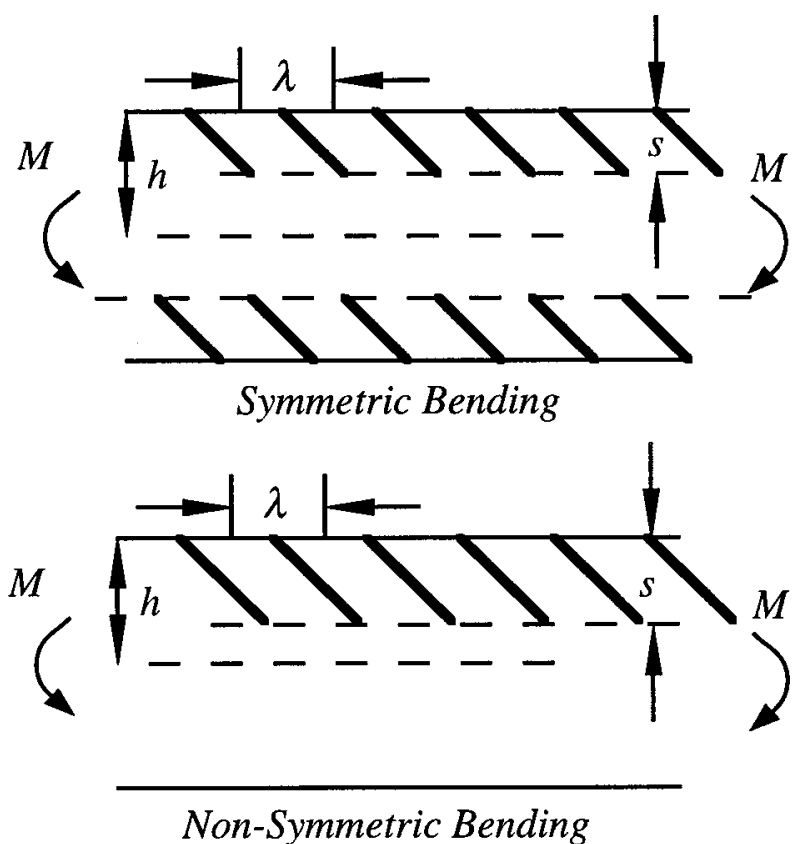

FIG. 6. Shear bands in a bent plate.

shear planes and to extend to the depth of the plastic zone $s$, as shown in the figure. The spacing of the shear bands $\lambda$ can be estimated by assuming that each of the shear bands is like a Mode II surface crack of length $a=s \sqrt{2}$. The shear offset in the band at the surface relaxes the strain in the vicinity of the shear band and this establishes the shear band spacing. We imagine that because of the local strain relaxation, another shear band cannot be created close to an existing one. This effect would cause the shear bands to be spaced according to their lengths.

The shear displacement discontinuity for a Mode II crack of length $2 a$, subjected to a remote shear stress $\tau$ and with a uniform shear resistance $\tau_{y}$ acting on the crack faces, may be expressed as

$$
\Delta u=\frac{(1-2 \nu)}{\mu}\left(\tau-\tau_{y}\right) \sqrt{a^{2}-x^{2}} .
$$

We now apply this to the shear bands. We take $\tau$ to be the shear stress acting on the shear band at the top surface of the plate. This is approximated as the shear stress that would act there if the plate were bending in an elastic manner. We also take $\tau_{y}$ to be the shear yield stress, $a=s \sqrt{2}$ to be the length of the shear band, and $x$ to be measured along the shear band from the surface. The maximum shear offset, which occurs at $x=0$, is then approximately

$$
\Delta u_{\max }=\frac{(1-2 \nu)}{\mu}\left(\tau-\tau_{y}\right) a .
$$

Taking $\tau=\sigma_{x x}(y=h) / 2$ (for elastic bending) and $\tau_{y}=\sigma_{y} / 2$, and using Hooke's law, we may write

$$
\Delta u_{\max }=\frac{(1-2 \nu)}{(1-\nu)}\left[\varepsilon_{x x}(y=h)-\varepsilon_{y}\right] a .
$$


If the shear offsets are assumed to elastically unload the material on either side of the shear bands, then the shear bands will be spaced apart in direct proportion to the shear offsets. Thus the shear band spacing $\lambda$ cannot be smaller than

$$
\lambda=\frac{\Delta u_{\max }}{\sqrt{2} \varepsilon_{x x}(y=h)} .
$$

Taking this to be the average shear band spacing and using Eq. (17) and $a=s \sqrt{2}$, we find

$$
\lambda=\frac{(1-2 \nu)}{(1-\nu)}\left(1-\frac{\varepsilon_{y}}{\varepsilon_{x x}(y=h)}\right) s .
$$

This may be expressed in terms of the curvatures as

$$
\lambda=\frac{(1-2 \nu)}{(1-\nu)}\left(1-\frac{\kappa_{y}}{\kappa}\right) s,(\text { symmetric bending }),
$$

where we have used Eq.(1) and

$$
\lambda=\left(\frac{1-2 \nu}{1-\nu}\right)\left(\frac{2 \frac{\kappa}{\kappa_{y}}\left(1-\sqrt{\frac{\kappa_{y}}{\kappa}}\right)}{1+2 \frac{\kappa}{\kappa_{y}}\left(1-\sqrt{\frac{\kappa_{y}}{\kappa}}\right)}\right) s,
$$

( nonsymmetric bending),

where we have used Eqs. (1)-(3) and Eq. (12). But since the plastic zone size $s$ depends on the imposed curvature and the plate thickness, through Eq. (6) (for symmetric bending) and Eq. (11) (for nonsymmetric bending), we have

$$
\lambda=\frac{(1-2 \nu)}{(1-\nu)}\left(1-\frac{\kappa_{y}}{\kappa}\right)^{2} h,(\text { symmetric bending }),
$$

and

$$
\lambda=2 h\left(\frac{1-2 \nu}{1-\nu}\right)\left(\frac{2 \frac{\kappa}{\kappa_{y}}\left(1-\sqrt{\frac{\kappa_{y}}{\kappa}}\right)^{2}}{1+2 \frac{\kappa}{\kappa_{y}}\left(1-\sqrt{\frac{\kappa_{y}}{\kappa}}\right)}\right),
$$

(nonsymmetric bending).

These relations show that the shear band spacing in bending is expected to scale with the thickness of the plate, $2 h$. Figure 3 shows that this kind of relation is very well obeyed for various metallic glasses. In order to compare the observed shear band spacings with the predictions of Eqs. (22) and (23), we need to determine the curvatures at which yielding and fracture occurs. Below we develop a model to estimate the sample size dependence of the shear band spacing for Vitreloy I at the point of fracture in bending.

\section{CRACK INITIATION AND GROWTH IN THE SHEAR BAND}

The analysis above suggests that as shear bands form at the surfaces of the plate during bending, the mean spacing increases. For $\kappa=\kappa_{y}$, for example, the shear band spacing is predicted by Eqs. (22) and (23) to be to zero; the spacing increases with increasing curvature beyond the point of yielding. We imagine that some shear bands extend during

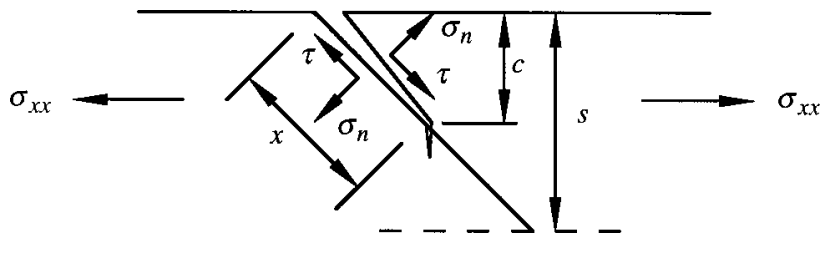

FIG. 7. Mixed mode crack of half length $x$ in a shear band.

bending while others do not, such that the mean spacing of the bands increases during the bending process. As the shear band structure coarsens with increased bending, the shear offsets in each shear band increase dramatically and cracks are eventually nucleated in the bands. Here we assume that when the shear offset in the band exceeds a critical value, $\Delta u^{*}$, the shear band is transformed into a mixed mode crack (Modes I and II). Setting the maximum shear offset given by Eq. (17) equal to $\Delta u^{*}$ and expressing the strains in terms of the corresponding curvatures allows one to determine the critical curvature $\kappa_{i}$, at which a crack begins to initiate (subscript $i$ for initiate) in the shear band. The results are

$$
\kappa_{i}=\kappa_{y}+\frac{\alpha}{2}\left(1+\sqrt{1+\frac{4 \kappa_{y}}{\alpha}}\right),(\text { symmetric bending })
$$

and

$$
\kappa_{i}=\kappa_{y}\left(1+\sqrt{\frac{\alpha}{4 \kappa_{y}}}\right)^{2},(\text { nonsymmetric bending }),
$$

where

$$
\alpha=\frac{(1-\nu)}{(1-2 \nu)} \frac{\Delta u^{*}}{\sqrt{2} h^{2}} .
$$

As the curvature increases beyond $\kappa_{i}$ the cracks increase in length as the displacement offsets in the shear bands exceed the critical value along the shear bands. Figure 7 shows a mixed mode crack that might grow by this process. Equation (15) for the shear displacement distribution in a shear band may be expressed in terms of the strains at the top surface of the plate as follows:

$$
\Delta u=\frac{(1-2 \nu)}{(1-\nu)}\left[\varepsilon_{x x}(y=h)-\varepsilon_{y}\right] \sqrt{a^{2}-x^{2}},
$$

where Hooke's law has been used.

For the case of symmetric bending, Eq. (27) together with Eq. (1) leads directly to

$$
\Delta u=\frac{(1-2 \nu)}{(1-\nu)}\left(\kappa-\kappa_{y}\right) h \sqrt{a^{2}-x^{2}} .
$$

Using $a=s \sqrt{2}$ and $x=c \sqrt{2}$, as illustrated in Fig. 5, and Eq. (6), we find

$$
\Delta u=\sqrt{2} \frac{(1-2 \nu)}{(1-\nu)}\left(\kappa-\kappa_{y}\right) h^{2} \sqrt{\left(1-\frac{\kappa_{y}}{\kappa}\right)^{2}-\left(\frac{c}{h}\right)^{2}},
$$

( symmetric bending). 
A corresponding expression for the case of nonsymmetric bending can be found by starting with Eq. (27), using Eq. (9) and Hooke's law to find

$$
\varepsilon_{x x}(y=h)-\varepsilon_{y}=\varepsilon_{y} \frac{4 h s}{(2 h-s)^{2}} .
$$

With Eq. (11) for $s$, we obtain,

$$
\Delta u=2 \frac{(1-2 \nu)}{(1-\nu)} \varepsilon_{y} \frac{\kappa}{\kappa_{y}}\left(1-\sqrt{\frac{\kappa_{y}}{\kappa}}\right) \sqrt{a^{2}-x^{2}} .
$$

Again using $a=s \sqrt{2}$ and $x=c \sqrt{2}$, as illustrated in Fig. 5, and Eq. (11), we find

$$
\begin{aligned}
\Delta u= & 4 \sqrt{2} \frac{(1-2 \nu)}{(1-\nu)} \kappa_{y} h^{2}\left(\frac{\kappa}{\kappa_{y}}-\sqrt{\frac{\kappa}{\kappa_{y}}}\right) \\
& \times \sqrt{\left(1-\frac{\kappa_{y}}{\kappa}\right)^{2}-\left(\frac{c}{2 h}\right)^{2}}
\end{aligned}
$$

(nonsymmetric bending).

Equations (29) and (32) give the shear displacements in the shear bands as a function of the loading and the perpendicular distance $c$ from the surface. By setting $\Delta u$ in Eqs. (29) and (32) equal to $\Delta u^{*}$ we can determine the length of the stable mixed mode crack forming in the shear band, for the cases of symmetric and nonsymmetric bending, respectively. The results are

$$
c=h \sqrt{\left(\frac{\kappa-\kappa_{y}}{\kappa}\right)^{2}-\left(\frac{\alpha}{\kappa-\kappa_{y}}\right)^{2}},
$$

(symmetric bending),

and

$$
c=2 h \sqrt{\left(1-\sqrt{\frac{\kappa_{y}}{\kappa}}\right)^{2}-\left(\frac{\frac{\alpha}{4 \kappa_{y}}}{\frac{\kappa}{\kappa_{y}}\left(1-\sqrt{\frac{\kappa_{y}}{\kappa}}\right)}\right)^{2}},
$$

(nonsymmetric bending),

where again $\alpha$ is given by Eq. (26). Setting $c=0$ in these equations and solving for $\kappa_{i}$ gives Eq. (24) for symmetric bending and Eq. (25) for nonsymmetric bending, as expected.

\section{UNSTABLE CRACK GROWTH AND FRACTURE}

We may now ask whether the mixed mode crack forming in the shear band will propagate in an unstable manner. It is easy to show that the crack extension force for crack extension perpendicular to the free surface (as shown in Fig. 7) is greater than the crack extension force for extension in the plane of the shear band. Thus, we will assume that unstable crack growth and fracture occurs when the stress intensity factor $K_{I}$ for a Mode I surface crack perpendicular to the surface exceeds the fracture toughness of the glass $K_{I_{c}}$. If the cracks in the shear bands are sufficiently far apart, the stress intensity factor for the case of plate bending may be expressed as ${ }^{15}$

$$
\begin{aligned}
K_{I}= & \frac{3 M}{2 h^{2}} \sqrt{\pi c}\left[1.12-1.39\left(\frac{c}{2 h}\right)+7.3\left(\frac{c}{2 h}\right)^{2}-13\left(\frac{c}{2 h}\right)^{3}\right. \\
& \left.+14\left(\frac{c}{2 h}\right)^{4}\right],
\end{aligned}
$$

where $M$ is the applied moment (per unit width), as described above. Using Eq. (7) for the moment $M$ and Eq. (8), the stress intensity factor may be expressed as

$$
\begin{aligned}
K_{I}= & {\left[\frac{2 \mu}{(1-\nu)} h \frac{\kappa_{y}^{3}}{\kappa^{2}}+\frac{3}{2} \sigma_{y}\left(1-\frac{\kappa_{y}^{2}}{\kappa^{2}}\right)\right] \sqrt{\pi c} * } \\
& \times\left[1.12-\frac{1.39}{2}\left(\frac{c}{h}\right)+\frac{7.3}{4}\left(\frac{c}{h}\right)^{2}-\frac{13}{8}\left(\frac{c}{h}\right)^{3}+\frac{14}{16}\left(\frac{c}{h}\right)^{4}\right],
\end{aligned}
$$

( symmetric bending),

for symmetric bending, and, using Eqs. (14) and (8), the stress intensity factor for nonsymmetric bending is

$$
\begin{aligned}
K_{I}= & \sigma_{y} \sqrt{\pi c}\left(3-2 \sqrt{\frac{\kappa_{y}}{\kappa}}\right) *\left(1.12-\frac{1.39}{2}\left(\frac{c}{h}\right)+\frac{7.3}{4}\left(\frac{c}{h}\right)^{2}\right. \\
& \left.-\frac{13}{8}\left(\frac{c}{h}\right)^{3}+\frac{14}{16}\left(\frac{c}{h}\right)^{4}\right),
\end{aligned}
$$

(nonsymmetric bending).

Inserting Eq. (33) into Eq. (36) for symmetric bending, and Eq. (34) into Eq. (37) for nonsymmetric bending, gives expressions for the Mode I stress intensity factor as a function of the bending curvature. Solving

$$
K_{I}=K_{I_{c}}
$$

for the curvature gives the critical curvature for unstable crack growth or fracture, $\kappa_{f}$. The progression of curvatures in bending must be such that the curvature needed to cause fracture is greater than that needed to initiate cracking in the shear band and that, in turn, must be greater than that needed to cause yielding: $\kappa_{f}>\kappa_{i}>\kappa_{y}$.

\section{EFFECT OF PLATE THICKNESS ON FRACTURE}

To study the effect of plate thickness on the fracture properties of metallic glass plates in bending, we calculate the stress intensity factors for shear band cracks in plates of different thickness and for different amounts of bending. It is convenient to use the strain on the surface of the plate $\varepsilon$ $=\varepsilon_{x x}(y=h)$ as a measure of bending in place of the curvature. With this replacement the stress intensity factor can be expressed as

$$
\begin{aligned}
K_{I}= & \left\{\frac{2 \mu}{(1-\nu)} \varepsilon_{y}\left[\frac{3}{2}-\frac{1}{2}\left(\frac{\varepsilon_{y}}{\varepsilon}\right)^{2}\right]\right\} \sqrt{\pi c} *\left[1.12-\frac{1.39}{2}\left(\frac{c}{h}\right)\right. \\
& \left.+\frac{7.3}{4}\left(\frac{c}{h}\right)^{2}-\frac{13}{8}\left(\frac{c}{h}\right)^{3}+\frac{14}{16}\left(\frac{c}{h}\right)^{4}\right]
\end{aligned}
$$


for symmetic bending, where the crack length is

$$
c=h \sqrt{\left[1-\left(\frac{\varepsilon_{y}}{\varepsilon}\right)\right]^{2}-\left(\frac{h \alpha}{\varepsilon-\varepsilon_{y}}\right)^{2}} .
$$

Equation (39) can be used provided the strain at the surface of the plate exceeds that needed to initiate cracks

$$
\varepsilon_{i}=\varepsilon_{y}+\frac{\alpha h}{2}\left(1+\sqrt{1+\frac{4 \varepsilon_{y}}{\alpha h}}\right) .
$$

For the case of nonsymmetic bending the stress intensity factor is

$$
\begin{aligned}
K_{I}= & {\left[\frac{2 \mu}{(1-\nu)} \varepsilon_{y}\left(3-\frac{4}{\left(1-\sqrt{\frac{2 \varepsilon_{y}}{\varepsilon}-1}\right)}\right)\right] \sqrt{\pi c^{*}} } \\
& \times\left[1.12-\frac{1.39}{2}\left(\frac{c}{h}\right)+\frac{7.3}{4}\left(\frac{c}{h}\right)^{2}-\frac{13}{8}\left(\frac{c}{h}\right)^{3}\right. \\
& \left.+\frac{14}{16}\left(\frac{c}{h}\right)^{4}\right],
\end{aligned}
$$

where the crack length is

$$
c=2 h \sqrt{\left(1-\sqrt{\frac{\varepsilon_{y}}{\varepsilon}}\right)^{2}-\left(\frac{\frac{\alpha h}{4 \varepsilon_{y}}}{\frac{\varepsilon}{\varepsilon_{y}}-\sqrt{\frac{\varepsilon}{\varepsilon_{y}}}}\right)^{2}} .
$$

Equation (42) is valid if the strain at the surface of the plate is greater than the critical strain needed for the initiation of cracks

$$
\varepsilon_{i}=\varepsilon_{y}\left(1+\sqrt{\frac{\alpha h}{4 \varepsilon_{y}}}\right)^{2} .
$$

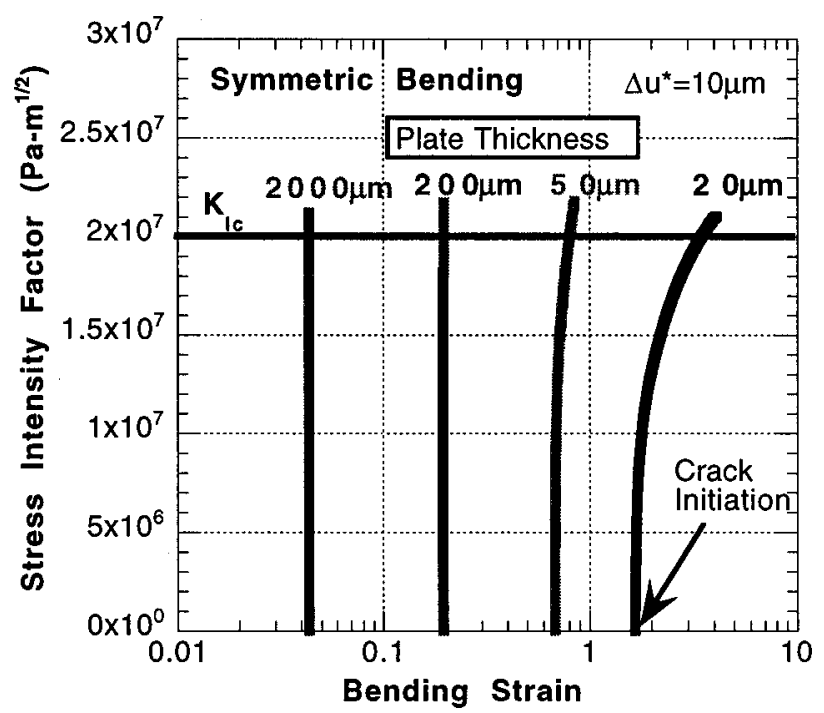

FIG. 8. Stress intensity factors for surface cracks in Vitreloy as a function of bending strain for different plate thicknesses, for the case of symmetric bending. The maximum bending strain is the strain at the surface of the plate. The fracture toughness of $K_{I_{c}}=20 \mathrm{MPa} \sqrt{m}$ is shown as a horizontal line in the figure. Intersection of the curves with the fracture toughness indicates the surface bending strain at fracture.
TABLE I. Mechanical properties of vitreloy I and model parameters.

\begin{tabular}{lcc}
\hline \hline Quantity & Symbol & Value \\
\hline Young's modulus & $\mathrm{E}$ & $97 \mathrm{GPa}$ \\
Shear modulus & $\mu$ & $35.7 \mathrm{GPa}$ \\
Poisson's ratio & $\nu$ & 0.36 \\
Yield strength & $\sigma_{y}$ & $1.9 \mathrm{GPa}$ \\
Yield strain & $\varepsilon_{y}$ & 0.017 \\
Critical shear offset & $\Delta u^{*}$ & $10 \mu \mathrm{m}$ \\
Fracture toughness & $K_{I c}$ & $20 \mathrm{Mpa} \mathrm{m}^{1 / 2}$ \\
\hline \hline
\end{tabular}

Figure 8 shows a plot of the stress intensity factor for shear band cracks as a function of bending strain for plates of different thickness, for the case of symmetric bending, using the elastic and plastic properties of Vitreloy I (shown in Table I) for purposes of analysis. The fracture toughness of Vitreloy I is also shown in the diagram as a horizontal line. The intersection of the curves with the fracture toughness indicates the bending strain at fracture. The figure shows the dramatic effect of plate thickness on the fracture strain.

The predicted bending strain at fracture is shown in Fig. 9 as a function of plate thickness for both symmetric and nonsymmetric bending. The dramatic effect of plate thickness is again observed. Above a plate thickness of about $1000 \mu \mathrm{m}=1 \mathrm{~mm}$, the plastic fracture strain (total strain minus the elastic strain) is about $1 \%$.

We showed in Fig. 3 that the shear band spacing for various metallic glasses subjected to bending depends linearly on the sample size. A relationship of that kind is a natural part of the present analysis. We showed in Eqs. (22) and (23) that the shear band spacing scales with the plate thickness. To examine this relation more closely we can calculate the shear band spacing at the point of fracture using the computed fracture strains (or corresponding curvatures) and Eqs. (22) and (23). Figure 10 shows the results of these calculations. There we see that the shear band spacing at the

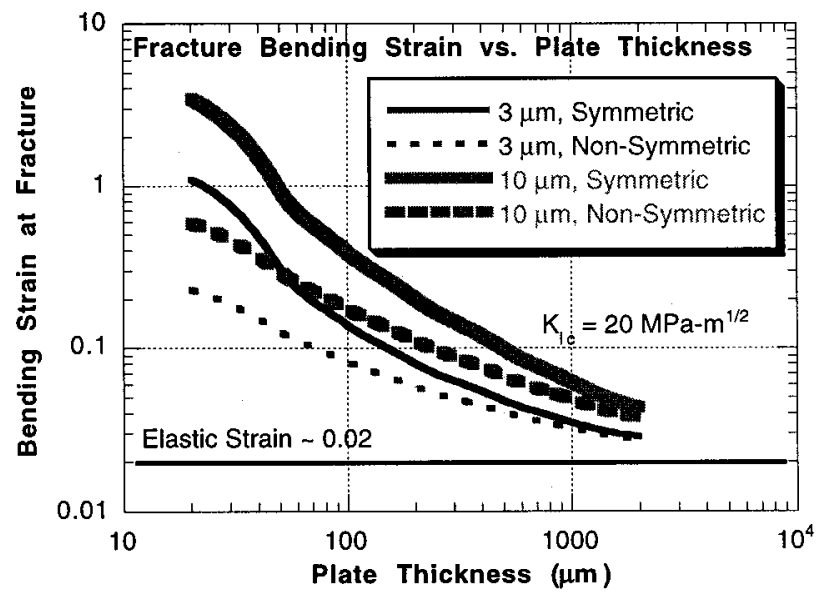

FIG. 9. Calculated fracture bending strain vs plate thickness for Vitreloy I for both symmetric and nonsymmetric bending for two different values of the critical shear displacement, $\Delta u^{*}, 3 \mu \mathrm{m}$, and $10 \mu \mathrm{m}$. A fracture toughness of $K_{I_{c}}=20 \mathrm{MPa} \sqrt{m}$ was used for these calculations. Above a plate thickness of about $1000 \mu \mathrm{m}=1 \mathrm{~mm}$ the plastic fracture strain (total strain minus the elastic strain) is about $1 \%$. These results are similar to the experimental results shown in Fig. 2. 


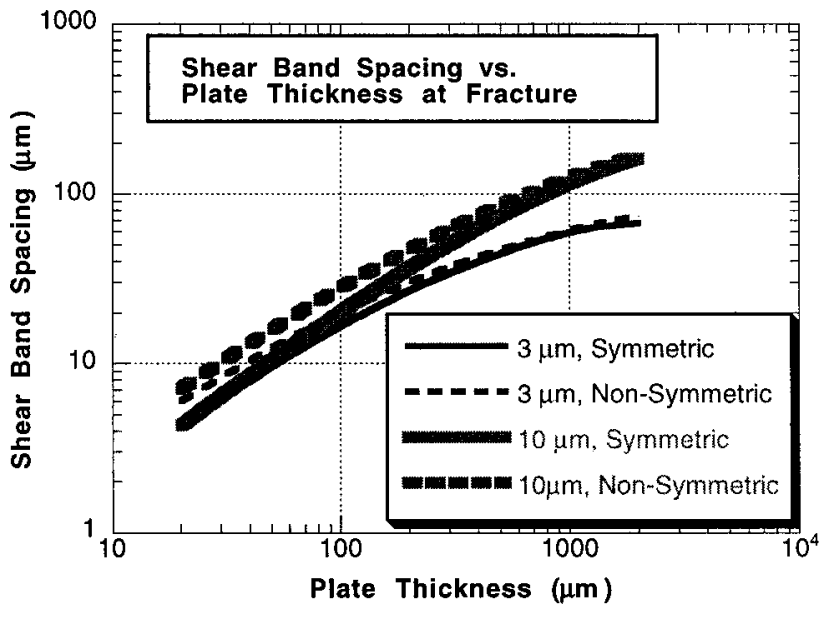

FIG. 10. Calculated shear band spacing vs plate thickness at the point of fracture for Vitreloy I for both symmetric and nonsymmetic bending for two different values of the critical shear displacement, $\Delta u^{*}, 3 \mu \mathrm{m}$, and $10 \mu \mathrm{m}$. A fracture toughness of $K_{I_{c}}=20 \mathrm{MPa} \sqrt{m}$ was used for these calculations. The shear band spacing at the point of fracture is observed to increase with increasing plate thickness; the relationship is similar to the experimental results shown in Fig. 3.

point of fracture is predicted to increase significantly with plate thickness, for both symmetric and nonsymmetric bending, in qualitative agreement with experiment.

\section{DISCUSSION}

In the present analysis we have tried to rationalize the observation that the shear band spacing in metallic glass plates subjected to bending scales with plate thickness and the related observation that the bend ductility increases dramatically with decreasing plate thickness. We have suggested that shear bands transform into surface cracks on bending and ultimately lead to fracture. Because the shear band spacing and length is naturally predicted to scale with plate thickness, it follows that the shear offsets in the shear bands also scales with plate thickness. On the assumption that crack nucleation and extension in the shear bands requires a critical shear displacement and that a critical crack length is need for unstable fracture, it follows that the bend ductility decreases sharply with increasing plate thickness, as observed experimentally.

These sample size effects on bend ductility suggest that amorphous metals in the form of densely packed wires or foils, which are subjected to bending on loading, might have much greater ductility and toughness than monolithic amorphous metals. If the thicknesses of such microstructural features are kept well below $1 \mathrm{~mm}$, the shear offsets in the shear bands might be insufficient to initiate cracks. These ideas suggest that foam microstructures might be particularly attractive for controlling fracture in metallic glasses. The structural elements comprising foam microstructures are naturally subjected to bending strains. If the elements or members are sufficiently thin, then they will exhibit substantial bend ductility and the entire structure will behave in a tough, ductile manner.

There are a number of deficiencies in the present model that should be pointed out. The predictions do not follow the experimental results very closely, especially for thick plates. At very large plate thicknesses, the shear band spacing is predicted to approach a constant value. This is caused by the assumption that the shear band spacing scales with the size of the plastic zones, which do not continue to grow in size with plate thickness at large plate thicknesses because fracture intervenes. The entire treatment is also only approximate in several places. First, the assumption that the shear bands extend to the depth of the plastic zone calculated using the continuum theory of an elastic, perfectly plastic solid is, of course, an approximation. Also, the estimates of the shear offsets in the shear bands are based on the stresses and elastic strains at the very surface of the plate; the bending stress gradient is not taken into account in these estimates. Finally, our treatment of stable crack growth in the shear bands following crack initiation ignores the stress redistribution that accompanies crack formation. This is probably the weakest point in the present analysis.

In spite of these obvious weaknesses, the present analysis does give a credible account of the effects of sample size on the bend ductility of metallic glass plates and it also describes the observed effects of sample size on shear band spacing. Perhaps more detailed analyses of these problems will lead to an even better picture of these phenomena, and ultimately, to a predictive model of deformation and fracture of thin sections of metallic glasses subjected to bending.

\section{ACKNOWLEDGMENTS}

The authors gratefully acknowledge financial support for this work from the Defense Advanced Research Projects Agency (DARPA), under ARO Contract No. DAAD 19-011-0525, and Dr. Leo Christodoulou as Program Manager. (Approved for Public Release, Distribution Unlimited. Case No. 41829). The assistance of Wendelin J. Wright of Stanford University in connection with the preparation of this manuscript is also much appreciated.

${ }^{1}$ N. L. Petouhoff, A. J. Ardell, and A. F. Jankowski, Acta Metall. Mater. 40(11), 3167 (1992).

${ }^{2}$ S. Suto, K. Matsuno, T. Sano, and K. Matsui, J. Mater. Process. Technol. 33(3), 215 (1992).

${ }^{3}$ A. Inoue, K. Amiya, I. Yoshi, H. M. Kimura, and T. Masumoto, Mater. Trans., JIM 35 (7), 485 (1994).

${ }^{4}$ A. Inoue, K. Amiya, A. Katsuya, and T. Masumoto, Mater. Trans., JIM 36(7), 858 (1995).

${ }^{5}$ E. P. Barth, F. Spaepen, R. Bye, and S. K. Das, Acta Mater. 45(1), 423 (1997).

${ }^{6}$ A. Katsuya, A. Inoue, and T. Masumoto, Mater. Sci. Eng., A 226, 104 (1997).

${ }^{7}$ A. Inoue and X. M. Wang, Acta Mater. 48(6), 1383 (2000).

${ }^{8}$ Y. Yokoyama, K. Yamano, K. Fukaura, H. Sunada, and A. Inoue, Mater. Trans., JIM 42(4), 623 (2001).

${ }^{9}$ A. Katuya, A. Inoue, and K. Amiya, Int. J. Rapid Solidf. 9(2), 137 (1996).

${ }^{10}$ A. Inoue, A. Katsuya, K. Amiya, and T. Masumoto, Mater. Trans., JIM 36(7), 802 (1995).

${ }^{11}$ R. D. Conner, Y. Li, and W. L. Johnson (unpublished).

${ }^{12}$ P. E. Donovan, Mater. Sci. Eng. 98, 487 (1988).

${ }^{13}$ P. E. Donovan, Acta Metall. 37(2), 445 (1989).

${ }^{14}$ Wendelin J. Wright, Ranjana Saha, and William D. Nix, Mater. Trans., JIM 42(4), 642 (2001).

${ }^{15}$ K. Hellan, Introduction to Fracture Mechanics (McGraw-Hill, New York, 1984), p. 244. 